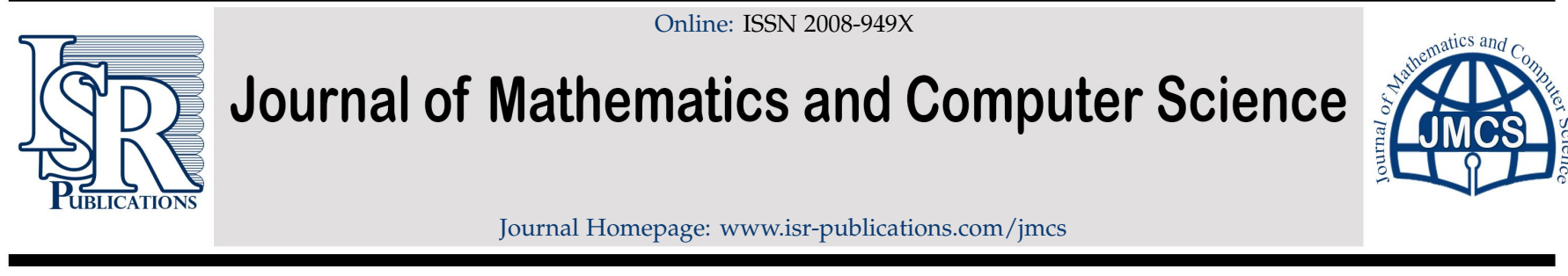

\title{
A study on r-edge regular intuitionistic fuzzy graphs
}

\author{
Ahmad Al-Jarrah a,*, Mourad Oqla Massa'deh ${ }^{\mathrm{a}}$, Abd el Karim Baareh ${ }^{\mathrm{a}, \mathrm{b}}$, Mohammad A. Aljarrah $^{\mathrm{c}}$, Roba \\ Mahmoud Alogla ${ }^{a}$, Nisrein Abu-Darwish ${ }^{a}$
}

${ }^{a}$ Applied Science Department, Ajloun University College, Al-Balqa Applied University, Ajloun 26816, Jordan.

${ }^{b}$ Department of Mathematics, Science College, Taibah University, Medina, Saudi Arabia.

${ }^{c}$ Department of Mathematics, Tafila Technical University, Tafila, Jordan.

\begin{abstract}
For an intuitionistic fuzzy set $S=\left\{<a, S_{\mu}(a), S_{\lambda}(a)>; a \in A\right\}$ of a universe set $A$, we discuss and study properties on an intuitionistic fuzzy graph like r-edge regular intuitionistic fuzzy graph and strongly regular intuitionistic fuzzy graph. In this paper, we describe variously related to these graphic's. Also, we have provided many examples to explain these concepts.
\end{abstract}

Keywords: Fuzzy set, intuitionistic fuzzy set, r-edge regular intuitionistic fuzzy graph, strongly edge regular, intuitionistic fuzzy graph, absolute degree of vertex.

2020 MSC: 03E72, 03F55, 54A40.

(C)2021 All rights reserved.

\section{Introduction}

In 1965, Zadeh [20] introduced a fuzzy set concept which led to change the science face and technology to a great extent. Since then, a fuzzy set theory has became a vigorous research area in different disciplines, like computer network, engineers, life sciences, mathematical modeling and medical science.

Rosenfeld [16] introduced fuzzy relations on fuzzy sets and generalized a fuzzy graph structure. Hereafter, several interesting results about fuzzy graphs and it's application have been obtained [6, 7, 11, 12, 15, 17]. Atanassov [1] in 1983 introduced intuitionistic fuzzzy set. An intuitionistic fuzzy set is characterized by two functions that expressing the membership degree and the non-membership degree of elements of the universe to intuitionistic fuzzy set. Research on intuitionistic fuzzy sets theory has been witnessing and exponential growth in mathematics and it's application. Karamambigai and Parvathi [13] studies intuitionistics fuzzy graph. The concepts of intuitionistics fuzzy graph and it's matrix was introduces by Karamambigai and Kalaivani [5]. In 2015, Pathinathan and Rosline [14] studied cartesian product of intuitionistic fuzzy graph and vertex degree, while Mishra and pal [8] discussed the concepts

\footnotetext{
${ }^{*}$ Corresponding author

Email addresses: aljarrah@bau.edu.jo (Ahmad Al-Jarrah), mourad.oqla@bau.edu.jo (Mourad Oqla Massa'deh), baareh@bau .edu .jo (Abd el Karim Baareh), aljarrah@ymail.com (Mohammad A. Aljarrah), robajabali@bau.edu.jo (Roba Mahmoud Alogla), nesreen.abudarwesh@bau.edu.jo (Nisrein Abu-Darwish)
}

doi: $10.22436 /$ jmcs.023.04.01

Received: 2020-08-23 Revised: 2020-10-07 Accepted: 2020-10-11 
of regular interval valued intuitionistic fuzzy graph. Various authors have discussed many properties and theorems for this concepts $[2-4,9,10,18,19]$.

In this paper, we study intuitionistic fuzzy graph with the help of some properties of r-edge regular and absolute degree of vertex. This paper is an attempt to generalize some concept from fuzzy graph to intuitionistic fuzzy graph.

\section{Preliminaries}

Definition 2.1 ([12]). A Graph $G=(V, E)$ is an ordered pair consisting of a non empty vertex set $V$, an edge set $E$ and a connection that associates with every edge between two edges called its end points.

Definition 2.2 ([12]). Let $G=(V, E)$ be a graph. Then $\delta_{G}=(A, B)$ is said to be subgraph of $G$ if $A \subseteq V$ and $\mathrm{B} \subseteq \mathrm{E}$.

Definition 2.3 ([20]). A fuzzy set $\mu$ on a set $A$ is just a function $\mu: A \rightarrow[0,1]$.

Definition 2.4 ([16]). A fuzzy graph $\delta_{G}=(V, \lambda, \mu)$ is a non empty set $V$ together with pair of functions $\lambda: V \rightarrow[0,1]$ and $\mu: V \times V \rightarrow[0,1]$ such that for all $a, b \in V, \mu(a b) \leqslant \min \{\lambda(a), \lambda(b)\}$ where $\lambda(a)$ and $\mu(a b)$ represent the membership values of the vertex $a$ and the edge $a b$ in $\delta_{G}$, respectively. The underlying crisp graph of the fuzzy graph $\delta_{G}=(V, \lambda, \mu)$ is denoted by $\delta_{G}{ }^{*}=\left(V, \lambda^{*}, \mu^{*}\right)$ where $\lambda^{*}=\{a \in V ; \lambda(a)>0\}$ and $\mu^{*}=\{a b \in V \times V ; \mu(a b)>0\}$. Thus for underlying fuzzy graph $\lambda^{*}=V$.

Definition 2.5 ([16]). A fuzzy graph $\delta_{G}=(V, \lambda, \mu)$ is said to be complete if $\mu(a b)=\min \{\lambda(a), \lambda(b)\}$ for all $a, b \in V$ where $a b$ denoted the edge between $a$ and $b$.

Definition 2.6 ([16]). A fuzzy graph $\delta_{S G}=\left(V, \lambda_{S}, \mu_{S}\right)$ is said to subgraph of $\delta_{G}=(V, \lambda, \mu)$ if $\lambda_{S}(a) \leqslant \lambda(a)$ and $\mu_{S}(a b) \leqslant \mu(a b)$ for all $a \in V$ and all edges ab.

Definition 2.7 ([13]). If $\mathrm{A}$ is a non-empty set, an intuitionistic fuzzy set $\mu$ of $\mathrm{A}$ is an object

$$
\delta=\left\{<a, \delta_{\mu}(a), \delta_{\lambda}(a)>, a \in A\right\},
$$

where $\delta_{\mu}: A \rightarrow[0,1]$ and $\delta_{\lambda}: A \rightarrow[0,1]$ define the degree of membership and the degree of nonmembership of the element $a \in A$, respectively, with $0 \leqslant \delta_{\mu}(a)+\delta_{\lambda}(a) \leqslant 1$ for all a in A.

Definition 2.8 ([13]). An intuitionistic fuzzy set $\delta$ in $\mathrm{V}$ is defined by

$$
\delta=\left(\delta_{\mu}, \delta_{\lambda}\right): \mathrm{V} \times \mathrm{V} \rightarrow[0,1] \times[0,1],
$$

an intuitionistic fuzzy relation on $V$ such that $\delta_{m} u(a, b) \in[0,1]$ and $\delta_{\lambda}(a, b) \in[0,1]$.

Definition 2.9 ([13]). Let $\gamma=\left(\gamma_{\mu}, \gamma_{\lambda}\right)$ be an intuitionistic fuzzy set on a set $\mathrm{V}$. If $\delta$ is an intuitionistic fuzzy relation on $\mathrm{V}$, then $\delta$ is called an intuitionistic fuzzy relation on $\gamma$ if

$$
\gamma_{\mu}(a b) \leqslant \min \left\{\delta_{\mu}(a), \delta_{\mu}(b)\right\}, \quad \gamma_{\lambda}(a b) \geqslant \max \left\{\delta_{\lambda}(a), \delta_{\lambda}(b)\right\}
$$

for all $\mathrm{ab}$ in $\mathrm{E}$.

Definition 2.10 ([13]). An intuitionistic fuzzy graph is a pair $G=(\delta, \gamma)$ of a graph $G^{*}=(V, E)$, where $\delta=\left(\delta_{\mu}, \delta_{\lambda}\right)$ is an intuitionistic fuzzy set on $\mathrm{V}$ and $\gamma=\left(\gamma_{\mu}, \gamma_{\lambda}\right)$ is an intuitionistic fuzzy relation on $\mathrm{E}$ such that

$$
\gamma_{\mu}(a b) \leqslant \min \left\{\delta_{\mu}(a), \delta_{\mu}(b)\right\}, \quad \gamma_{\lambda}(a b) \geqslant \max \left\{\delta_{\lambda}(a), \delta_{\lambda}(b)\right\}
$$

for all $a, b$ in $V$. The underlying crisp graph of $G=(\delta, \gamma)$ is the crisp graph $G^{*}=(V, E)$, where

$$
V=\left\{a ; \delta_{\lambda}(a)>0 \text { or } \delta_{\lambda}(a)=0\right\} \text { and } E=\left\{a b ; \gamma_{\mu}(a b)>0 \text { or } \gamma_{\lambda}(a b)=0\right\} \text {. }
$$

Example 2.11. Consider an intuitionistic fuzzy graph $G$, such that

$$
V=\left\{a_{1}, a_{2}, a_{3}, a_{4}, a_{5}\right\} \text { and } E=\left\{a_{1} a_{2}, a_{2} a_{3}, a_{3} a_{4}, a_{4} a_{5}, a_{1} a_{3}, a_{3} a_{5}, a_{2} a_{4}\right\}
$$

as in Figure 1. 


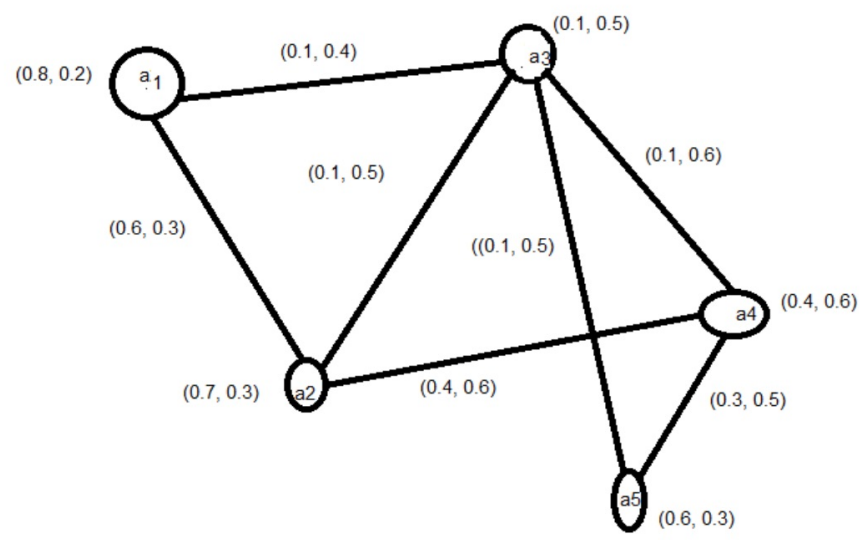

Figure 1: An example of an intuitionistic fuzzy graph.

\section{Intuitionistic fuzzy graph in r-edge regular}

Definition 3.1. An intuitionistic fuzzy graph $G=(\delta, \lambda)$ of a graph $G^{*}=(V, E)$ is said to be strong if

$$
\gamma_{\mu}(a b)=\min \left\{\delta_{\mu}(a), \delta_{\mu}(b)\right\}, \quad \gamma_{\lambda}(a b)=\max \left\{\delta_{\lambda}(a), \delta_{\lambda}(b)\right\}
$$

for all $a b \in E$.

Definition 3.2. An intuitionistic fuzzy graph $G=(\delta, \gamma)$ of a graph $G^{*}=(V, E)$ is said to be complete if

$$
\gamma_{\mu}(a b)=\min \left\{\delta_{\mu}(a), \delta_{\mu}(b)\right\}, \quad \gamma_{\lambda}(a b)=\max \left\{\delta_{\lambda}(a), \delta_{\lambda}(b)\right\}
$$

for all $a, b \in V$.

Definition 3.3. A complement of an intuitionistic fuzzy graph $G=(\delta, \gamma)$ is an intuitionistic fuzzy graph $\mathrm{G}^{\mathrm{c}}=\left(\delta^{\mathrm{c}}, \gamma^{\mathrm{c}}\right)$, where

$$
\delta^{c}=\delta=\left(\delta_{\mu}(a), \delta_{\lambda}(a)\right) \text { and } \gamma^{c}=\left(\gamma_{\mu}^{c}(a), \gamma_{\lambda}^{c}(a)\right)
$$

here

$$
\gamma_{\mu}^{c}(a b)=\min \left\{\delta_{\mu}(a), \delta_{\mu}(b)\right\}-\gamma_{\mu}(a b), \quad \gamma_{\lambda}^{c}(a b)=\max \left\{\delta_{\lambda}(a), \delta_{\lambda}(b)\right\}-\gamma_{\lambda}(a b)
$$

for all $a b \in E$.

Definition 3.4. If $G=(\delta, \gamma)$ is an intuitionistic fuzzy graph, then the absolute degree of any vertex a is defined by

$$
D_{a}=\left(\mu_{D}(a), \lambda_{D}(a)\right),
$$

where

$$
\mu_{D}(a)=\sum \gamma_{\mu}(a b) ; a \neq b, a b \in E, \quad \text { and } \quad \lambda_{D}(a)=\sum \gamma_{\lambda}(a b) ; a \neq b, a b \in E,
$$

and hence

$$
D_{a}=\left|\sum_{\substack{a \neq b \\ a \in V}} \gamma_{\mu}(a b)-\sum \gamma_{\lambda}(a b)\right| .
$$

Example 3.5. Let $\mathrm{G}^{*}=(\mathrm{V}, \mathrm{E})$, where $\mathrm{V}=\{\mathrm{a}, \mathrm{b}, \mathrm{c}, \mathrm{d}, \mathrm{e}\}$ and $\mathrm{E}=\{\mathrm{ab}$, ae, bc, bd, $\mathrm{cd}$, de $\}$ (Figure 2), then

$$
\begin{aligned}
& \mathrm{D}_{\mathrm{a}}=|(0.1+0.1)-(0.5+0.5)|=0.8, \\
& \mathrm{D}_{\mathrm{b}}=|(0.1+0.1+0.3)-(0.5+0.5+0.5)|=1, \\
& \mathrm{D}_{\mathrm{c}}=|(0.3+0.3)-(0.2+0.4)|=0, \\
& \mathrm{D}_{\mathrm{d}}=\mid(0.3+0.2+0.3)-(0.2+0.6+0.5 \mid=0.5, \\
& \mathrm{D}_{e}=|(0.2+0.1)-(0.6+0.5)|=0.8 .
\end{aligned}
$$




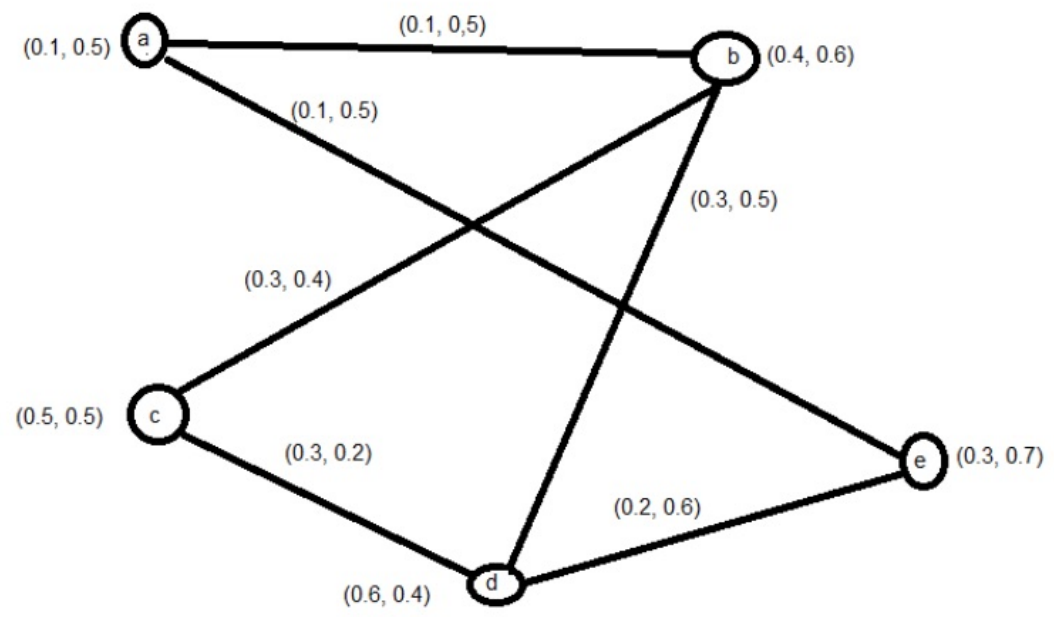

Figure 2: graph $\mathrm{G}^{*}$ which is defined in Example 3.5.

Definition 3.6. Let $\mathrm{G}^{*}=(\mathrm{V}, \mathrm{E})$ be a crisp graph and $\mathrm{i}=\mathrm{ab}$ be an edge in $\mathrm{G}^{*}$, then the degree of an edge $e \in E$ is defined as $D_{e}=D_{a}+D_{b}-2$.

Definition 3.7. A minimum open neighborhood degree of an edge is defined as $\Delta_{E}=\min \left\{D_{a b} ; a b \in E\right\}$ and the maximum open neighborhood degree of an edge is defined as $\Delta_{E}=\max \left\{D_{a b} ; a b \in E\right\}$.

Definition 3.8. The total open neighborhood degree of an edge $a b \in E$ in an intuitionistic fuzzy graph $\mathrm{G}=(\delta, \gamma)$ defined as

$$
\mathrm{T}_{\mathrm{D}}(\mathrm{ab})=\left(\mathrm{T}_{\mathrm{D}} \delta_{\mu}(\mathrm{ab}), \mathrm{T}_{\mathrm{D}} \lambda \mu(\mathrm{ab})\right),
$$

where

$$
\mathrm{T}_{\mathrm{D}} \delta \mu(\mathrm{ab})=\mu_{\mathrm{D}}(\mathrm{a})+\mu_{\mathrm{D}}(\mathrm{b})-\gamma_{\mu}(\mathrm{ab}), \quad \mathrm{T}_{\mathrm{D}} \lambda_{\mu}(\mathrm{ab})=\lambda_{\mathrm{D}}(\mathrm{a})+\lambda_{\mathrm{D}}(\mathrm{b})-\gamma_{\mu}(\mathrm{ab}) .
$$

The minimum total open neighborhood degree of an edge is defined as

$$
\Delta_{\mathrm{TE}}=\min \left\{\mathrm{T}_{\mathrm{D}}(\mathrm{ab}) ; \mathrm{ab} \in \mathrm{E}\right\}
$$

and the maximum open neighborhood degree of an edge is defined as

$$
\Delta_{\mathrm{TE}}=\max \left\{\mathrm{T}_{\mathrm{D}}(\mathrm{ab}) ; \mathrm{ab} \in \mathrm{E}\right\} .
$$

Definition 3.9. Let $\mathrm{G}=(\delta, \gamma)$ be an intuitionistic fuzzy graph. The degree neighborhood of a vertex a is defined as $D_{N}(a)=\left(N_{\delta}(a), N_{\gamma}(a)\right)$, where

$$
N_{\delta}(a)=\sum_{b \in N(a)} \delta_{\lambda}(b) \text { and } N_{\gamma}(a)=\sum_{b \in N(a)} \delta_{\mu}(b)
$$

Definition 3.10. Let $G=(\delta, \gamma)$ be an intuitionistic fuzzy graph on $G^{*}$, then

1. if all edges have the same neighborhood degree $r$, then $G$ is said to be r-edge regular intuitionistic fuzzy graph;

2. the open neighborhood degree of an edge $a b \in E$ in an intuitionistic fuzzy graph $G=(\delta, \gamma)$ is defined as $D_{a b}=\left(D_{\delta \mu}(a b), D_{\delta \lambda}(a b)\right)$ such that:

$$
D_{\delta \mu}(a b)=\mu_{D}(a)+\mu_{D}(b)-2 \gamma_{\mu}(a b), \quad D_{\delta \lambda}(a b)=\lambda_{D}(a)+\lambda_{D}(b)-2 \gamma_{\lambda}(a b) .
$$


Example 3.11. Consider an intuitionistic fuzzy graph $G=(\delta, \gamma)$ such that

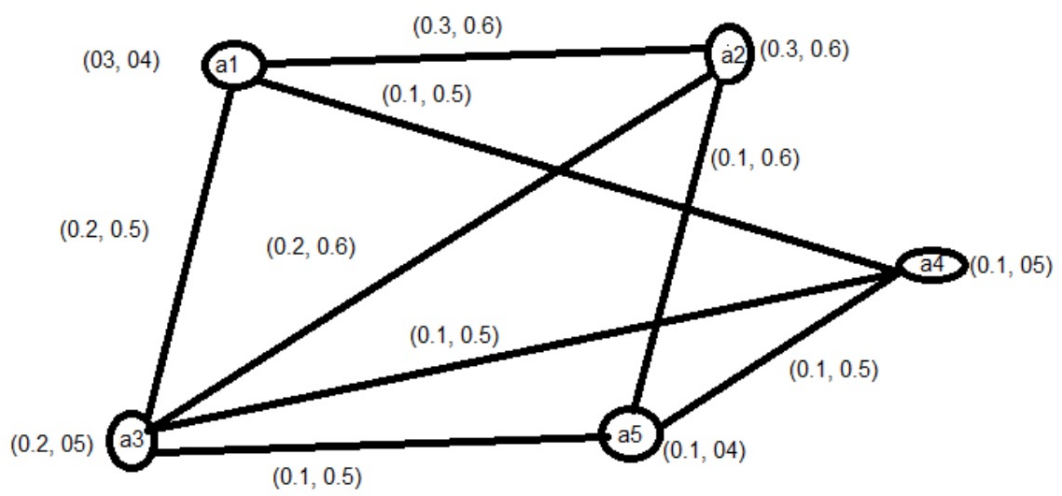

Figure 3: An example of an intuitionistic fuzzy graph.

$$
\begin{aligned}
& \mathrm{D}_{\mathrm{a}_{1}}=(0.6,1.6), \quad \mathrm{D}_{\mathrm{a}_{2}}=(0.6,1.7), \quad \mathrm{D}_{\mathrm{a}_{3}}=(0.5,2.1), \\
& \mathrm{D}_{\mathrm{a}_{4}}=(0.3,1.6), \quad \mathrm{D}_{\mathrm{a}_{5}}=(0.3,1.6) \text {, } \\
& D_{\delta \mu}\left(a_{1} a_{2}\right)=\mu_{D}\left(a_{1}\right)+\mu_{D}\left(a_{2}\right)-2 \gamma_{\mu}\left(a_{1} a_{2}\right)=0.6+0.6-2(0.3)=0.6 \text {, } \\
& D_{\delta \lambda}\left(a_{1} a_{2}\right)=\lambda_{D}\left(a_{1}\right)+\lambda_{D}\left(a_{2}\right)-2 \gamma_{\lambda}\left(a_{1} a_{2}\right)=1.6+2.1-2(0.6)=2.6 \text {, } \\
& D_{a_{1} a_{2}}=\left(D_{\delta \mu}\left(a_{1} a_{2}\right), D_{\delta \lambda}\left(a_{1} a_{2}\right)\right)=(0.6,2.6), \\
& D_{\delta \mu}\left(a_{1} a_{3}\right)=\mu_{D}\left(a_{1}\right)+\mu_{D}\left(a_{3}\right)-2 \gamma_{\mu}\left(a_{1} a_{3}\right)=0.6+0.6-2(0.2)=0.8 \text {, } \\
& D_{\delta \lambda}\left(a_{1} a_{3}\right)=\lambda_{D}\left(a_{1}\right)+\lambda_{D}\left(a_{3}\right)-2 \gamma_{\lambda}\left(a_{1} a_{3}\right)=1.6+2.1-2(0.5)=2.7 \text {, } \\
& D_{a_{1} a_{3}}=\left(D_{\delta \mu}\left(a_{1} a_{3}\right), D_{\delta \lambda}\left(a_{1} a_{3}\right)\right)=(0.8,2.7), \\
& D_{\delta \mu}\left(a_{1} a_{4}\right)=\mu_{D}\left(a_{1}\right)+\mu_{D}\left(a_{4}\right)-2 \gamma_{\mu}\left(a_{1} a_{4}\right)=0.6+0.3-2(0.1)=0.7 \text {, } \\
& D_{\delta \lambda}\left(a_{1} a_{4}\right)=\lambda_{D}\left(a_{1}\right)+\lambda_{D}\left(a_{4}\right)-2 \gamma_{\lambda}\left(a_{1} a_{4}\right)=1.6+1.6-2(0.5)=2.2 \text {, } \\
& D_{a_{1} a_{4}}=\left(D_{\delta \mu}\left(a_{1} a_{4}\right), D_{\delta \lambda}\left(a_{1} a_{4}\right)\right)=(0.7,2.2), \\
& D_{\delta \mu}\left(a_{2} a_{3}\right)=\mu_{D}\left(a_{2}\right)+\mu_{D}\left(a_{3}\right)-2 \gamma_{\mu}\left(a_{2} a_{3}\right)=0.6+0.5-2(0.1)=0.9 \text {, } \\
& D_{\delta \lambda}\left(a_{2} a_{3}\right)=\lambda_{D}\left(a_{2}\right)+\lambda_{D}\left(a_{3}\right)-2 \gamma_{\lambda}\left(a_{2} a_{3}\right)=1.7+2.1-2(0.6)=2.7 \text {, } \\
& D_{a_{2} a_{3}}=\left(D_{\delta \mu}\left(a_{2} a_{3}\right), D_{\delta \lambda}\left(a_{2} a_{3}\right)\right)=(0.9,2.7), \\
& D_{\delta \mu}\left(a_{2} a_{5}\right)=\mu_{D}\left(a_{2}\right)+\mu_{D}\left(a_{5}\right)-2 \gamma_{\mu}\left(a_{2} a_{5}\right)=0.6+0.3-2(0.1)=0.7 \text {, } \\
& D_{\delta \lambda}\left(a_{2} a_{5}\right)=\lambda_{D}\left(a_{2}\right)+\lambda_{D}\left(a_{5}\right)-2 \gamma_{\lambda}\left(a_{2} a_{5}\right)=1.7+1.6-2(0.6)=2.1 \text {, } \\
& D_{a_{2} a_{5}}=\left(D_{\delta \mu}\left(a_{2} a_{5}\right), D_{\delta \lambda}\left(a_{2} a_{5}\right)\right)=(0.7,2.1), \\
& D_{\delta \mu}\left(a_{3} a_{4}\right)=\mu_{D}\left(a_{3}\right)+\mu_{D}\left(a_{4}\right)-2 \gamma_{\mu}\left(a_{3} a_{4}\right)=0.6+0.3-2(0.1)=0.7 \text {, } \\
& D_{\delta \lambda}\left(a_{2} a_{4}\right)=\lambda_{D}\left(a_{2}\right)+\lambda_{D}\left(a_{4}\right)-2 \gamma_{\lambda}\left(a_{2} a_{4}\right)=2.1+1.6-2(0.5)=2.7 \text {, } \\
& D_{a_{3} a_{4}}=\left(D_{\delta \mu}\left(a_{3} a_{4}\right), D_{\delta \lambda}\left(a_{3} a_{4}\right)\right)=(0.7,2.7), \\
& D_{\delta \mu}\left(a_{3} a_{5}\right)=\mu_{D}\left(a_{3}\right)+\mu_{D}\left(a_{5}\right)-2 \gamma_{\mu}\left(a_{3} a_{5}\right)=0.6+0.3-2(0.1)=0.7 \text {, } \\
& D_{\delta \lambda}\left(a_{3} a_{5}\right)=\lambda_{D}\left(a_{3}\right)+\lambda_{D}\left(a_{5}\right)-2 \gamma_{\lambda}\left(a_{3} a_{5}\right)=2.1+1.6-2(0.5)=2.7 \text {, } \\
& D_{a_{3} a_{5}}=\left(D_{\delta \mu}\left(a_{3} a_{5}\right), D \delta \lambda\left(a_{3} a_{5}\right)\right)=(0.7,2.7), \\
& D_{\delta \mu}\left(a_{4} a_{5}\right)=\mu_{D}\left(a_{4}\right)+\mu_{D}\left(a_{5}\right)-2 \gamma_{\mu}\left(a_{4} a_{5}\right)=0.3+0.3-2(0.1)=0.4 \text {, } \\
& D_{\delta \lambda}\left(a_{4} a_{5}\right)=\lambda_{D}\left(a_{4}\right)+\lambda_{D}\left(a_{5}\right)-2 \gamma_{\lambda}\left(a_{4} a_{5}\right)=1.6+1.6-2(0.5)=2.2 \text {, } \\
& D_{a_{4} a_{5}}=\left(D_{\delta \mu}\left(a_{4} a_{5}\right), D_{\delta \lambda}\left(a_{4} a_{5}\right)\right)=(0.4,2.2), \\
& \mathrm{T}_{\mathrm{D}} \delta_{\mu}\left(\mathrm{a}_{1} \mathrm{a}_{2}\right)=\mu_{\mathrm{D}}\left(\mathrm{a}_{1}\right)+\mu_{\mathrm{D}}\left(\mathrm{a}_{2}\right)-\gamma_{\mu}\left(\mathrm{a}_{1} \mathrm{a}_{2}\right)=0.6+0.6-0.3=0.9 \text {, } \\
& \mathrm{T}_{\mathrm{D}} \lambda_{\mu}\left(\mathrm{a}_{1} \mathrm{a}_{2}\right)=\lambda_{\mathrm{D}}\left(\mathrm{a}_{1}\right)+\lambda_{\mathrm{D}}\left(\mathrm{a}_{2}\right)-\gamma_{\lambda}\left(\mathrm{a}_{1} \mathrm{a}_{2}\right)=1.6+2.1-0.6=3.1 \text {, }
\end{aligned}
$$




$$
\begin{aligned}
& T_{D}\left(a_{1} a_{2}\right)=\left(T_{D} \delta_{\mu}\left(a_{1} a_{2}\right), T_{D} \lambda_{\mu}\left(a_{1} a_{2}\right)\right)=(0.9,3.1), \\
& \mathrm{T}_{\mathrm{D}} \delta_{\mu}\left(\mathrm{a}_{1} \mathrm{a}_{3}\right)=\mu_{\mathrm{D}}\left(\mathrm{a}_{1}\right)+\mu_{\mathrm{D}}\left(\mathrm{a}_{3}\right)-\gamma_{\mathrm{m}} u\left(\mathrm{a}_{1} \mathrm{a}_{3}\right)=0.6+0.6-0.2=1 \text {, } \\
& \mathrm{T}_{\mathrm{D}} \lambda_{\mu}\left(\mathrm{a}_{1} \mathrm{a}_{3}\right)=\lambda_{\mathrm{D}}\left(\mathrm{a}_{1}\right)+\lambda_{\mathrm{D}}\left(\mathrm{a}_{3}\right)-\gamma_{\lambda}\left(\mathrm{a}_{1} \mathrm{a}_{3}\right)=1.6+2.1-0.5=3.2 \text {, } \\
& \mathrm{T}_{\mathrm{D}}\left(\mathrm{a}_{1} \mathrm{a}_{3}\right)=\left(\mathrm{T}_{\mathrm{D}} \delta_{\mu}\left(\mathrm{a}_{1} \mathrm{a}_{3}\right), \mathrm{T}_{\mathrm{D}} \lambda_{\mu}\left(\mathrm{a}_{1} \mathrm{a}_{3}\right)\right)=(1,3.2), \\
& \mathrm{T}_{\mathrm{D}} \delta_{\mu}\left(\mathrm{a}_{1} \mathrm{a}_{4}\right)=\mu_{\mathrm{D}}\left(\mathrm{a}_{1}\right)+\mu_{\mathrm{D}}\left(\mathrm{a}_{4}\right)-\gamma_{\mu}\left(\mathrm{a}_{1} \mathrm{a}_{4}\right)=0.6+0.3-0.1=0.8 \text {, } \\
& \mathrm{T}_{\mathrm{D}} \lambda_{\mu}\left(\mathrm{a}_{1} \mathrm{a}_{4}\right)=\lambda_{\mathrm{D}}\left(\mathrm{a}_{1}\right)+\lambda_{\mathrm{D}}\left(\mathrm{a}_{4}\right)-\gamma_{\lambda}\left(\mathrm{a}_{1} \mathrm{a}_{4}\right)=1.6+1.6-0.5=2.7 \text {, } \\
& T_{D}\left(a_{1} a_{4}\right)=\left(T_{D} \delta_{\mu}\left(a_{1} a_{4}\right), T_{D} \lambda_{\mu}\left(a_{1} a_{4}\right)\right)=(0.8,2.7), \\
& \mathrm{T}_{\mathrm{D}} \delta_{\mu}\left(\mathrm{a}_{2} \mathrm{a}_{3}\right)=\mu_{\mathrm{D}}\left(\mathrm{a}_{2}\right)+\mu_{\mathrm{D}}\left(\mathrm{a}_{3}\right)-\gamma_{\mu}\left(\mathrm{a}_{2} \mathrm{a}_{3}\right)=0.6+0.5-0.1=1 \text {, } \\
& \mathrm{T}_{\mathrm{D}} \lambda_{\mu}\left(\mathrm{a}_{2} \mathrm{a}_{3}\right)=\lambda_{\mathrm{D}}\left(\mathrm{a}_{2}\right)+\lambda_{\mathrm{D}}\left(\mathrm{a}_{3}\right)-\gamma_{\lambda}\left(\mathrm{a}_{2} \mathrm{a}_{3}\right)=1.7+2.1-0.6=3.2 \text {, } \\
& \mathrm{T}_{\mathrm{D}}\left(\mathrm{a}_{2} \mathrm{a}_{3}\right)=\left(\mathrm{T}_{\mathrm{D}} \delta_{\mu}\left(\mathrm{a}_{2} \mathrm{a}_{3}\right), \mathrm{T}_{\mathrm{D}} \lambda_{\mu}\left(\mathrm{a}_{2} \mathrm{a}_{3}\right)\right)=(1,3.2), \\
& \mathrm{T}_{\mathrm{D}} \delta_{\mu}\left(\mathrm{a}_{2} \mathrm{a}_{5}\right)=\mu_{\mathrm{D}}\left(\mathrm{a}_{2}\right)+\mu_{\mathrm{D}}\left(\mathrm{a}_{5}\right)-\gamma_{\mu}\left(\mathrm{a}_{2} \mathrm{a}_{5}\right)=0.6+0.3-0.1=0.8 \text {, } \\
& \mathrm{T}_{\mathrm{D}} \lambda_{\mu}\left(\mathrm{a}_{2} \mathrm{a}_{5}\right)=\lambda_{\mathrm{D}}\left(\mathrm{a}_{2}\right)+\lambda_{\mathrm{D}}\left(\mathrm{a}_{5}\right)-\gamma_{\lambda}\left(\mathrm{a}_{2} \mathrm{a}_{5}\right)=1.7+1.6-0.6=2.7 \text {, } \\
& \mathrm{T}_{\mathrm{D}}\left(\mathrm{a}_{2} \mathrm{a}_{5}\right)=\left(\mathrm{T}_{\mathrm{D}} \delta_{\mu}\left(\mathrm{a}_{2} \mathrm{a}_{5}\right), \mathrm{T}_{\mathrm{D}} \lambda_{\mu}\left(\mathrm{a}_{2} \mathrm{a}_{5}\right)\right)=(0.8,2.7), \\
& \mathrm{T}_{\mathrm{D}} \delta_{\mu}\left(\mathrm{a}_{3} \mathrm{a}_{4}\right)=\mu_{\mathrm{D}}\left(\mathrm{a}_{3}\right)+\mu_{\mathrm{D}}\left(\mathrm{a}_{4}\right)-\gamma_{\mu}\left(\mathrm{a}_{3} \mathrm{a}_{4}\right)=0.6+0.3-0.1=0.8 \text {, } \\
& \mathrm{T}_{\mathrm{D}} \lambda_{\mu}\left(\mathrm{a}_{3} \mathrm{a}_{4}\right)=\lambda_{\mathrm{D}}\left(\mathrm{a}_{3}\right)+\lambda_{\mathrm{D}}\left(\mathrm{a}_{4}\right)-\gamma_{\lambda}\left(\mathrm{a}_{3} \mathrm{a}_{4}\right)=2.1+1.6-0.5=3.2 \text {, } \\
& \mathrm{T}_{\mathrm{D}}\left(\mathrm{a}_{3} \mathrm{a}_{4}\right)=\left(\mathrm{T}_{\mathrm{D}} \delta_{\mu}\left(\mathrm{a}_{3} \mathrm{a}_{4}\right), \mathrm{T}_{\mathrm{D}} \lambda_{\mu}\left(\mathrm{a}_{3} \mathrm{a}_{4}\right)\right)=(0.8,2.7) \text {, } \\
& \mathrm{T}_{\mathrm{D}} \delta_{\mu}\left(\mathrm{a}_{3} \mathrm{a}_{5}\right)=\mu_{\mathrm{D}}\left(\mathrm{a}_{3}\right)+\mu_{\mathrm{D}}\left(\mathrm{a}_{5}\right)-\gamma_{\mu}\left(\mathrm{a}_{3} \mathrm{a}_{5}\right)=0.6+0.3-0.1=0.8 \text {, } \\
& \mathrm{T}_{\mathrm{D}} \lambda_{\mu}\left(\mathrm{a}_{3} \mathrm{a}_{5}\right)=\lambda_{\mathrm{D}}\left(\mathrm{a}_{3}\right)+\lambda_{\mathrm{D}}\left(\mathrm{a}_{5}\right)-\gamma_{\lambda}\left(\mathrm{a}_{3} \mathrm{a}_{5}\right)=2.1+1.6-0.5=3.2 \text {, } \\
& \mathrm{T}_{\mathrm{D}}\left(\mathrm{a}_{3} \mathrm{a}_{5}\right)=\left(\mathrm{T}_{\mathrm{D}} \delta_{\mu}\left(\mathrm{a}_{3} \mathrm{a}_{5}\right), \mathrm{T}_{\mathrm{D}} \lambda_{\mu}\left(\mathrm{a}_{3} \mathrm{a}_{5}\right)\right)=(0.8,2.7), \\
& \mathrm{T}_{\mathrm{D}} \delta_{\mu}\left(\mathrm{a}_{4} \mathrm{a}_{5}\right)=\mu_{\mathrm{D}}\left(\mathrm{a}_{4}\right)+\mu_{\mathrm{D}}\left(\mathrm{a}_{5}\right)-\gamma_{\mu}\left(\mathrm{a}_{4} \mathrm{a}_{5}\right)=0.3+0.3-0.1=0.5 \text {, } \\
& \mathrm{T}_{\mathrm{D}} \lambda_{\mu}\left(\mathrm{a}_{4} \mathrm{a}_{5}\right)=\lambda_{\mathrm{D}}\left(\mathrm{a}_{4}\right)+\lambda_{\mathrm{D}}\left(\mathrm{a}_{5}\right)-\gamma_{\lambda}\left(\mathrm{a}_{4} \mathrm{a}_{5}\right)=1.6+1.6-0.5=2.7 \text {, } \\
& T_{D}\left(a_{4} a_{5}\right)=\left(T_{D} \delta_{\mu}\left(a_{4} a_{5}\right), T_{D} \lambda_{\mu}\left(a_{4} a_{5}\right)\right)=(0.5,2.7) .
\end{aligned}
$$

Definition 3.12. Let $G=(\delta, \gamma)$ be an intuitionistic fuzzy graph. If every vertex in $G$ has the same degree $\left(r_{1}, r_{2}\right)$, then $G$ is said to be regular intuitionistic fuzzy graph of degree $\left(r_{1}, r_{2}\right)$.

Definition 3.13. In an intuitionistic fuzzy graph $G=(\delta, \gamma)$ if every edge has the same degree $\left(r_{1}, r_{2}\right)$, then $\mathrm{G}$ is said to be $\left(r_{1}, r_{2}\right)$-edge regular intuitionistic fuzzy graph.

Definition 3.14. If every edge in an intuitionistic fuzzy graph $G$ has the same total degree $\left(r_{1}, r_{2}\right)$, then $G$ is said to be a totally $\left(r_{1}, r_{2}\right)$-edge regular intuitionistic fuzzy graph.

Theorem 3.15. If $\mathrm{G}$ is an edge regular intuitionistic fuzzy graph on a cycle $\mathrm{G}^{*}$, then

$$
\sum_{a_{i} a_{j} \in E} D_{a_{i} a_{j}}=\sum_{a_{i} \in V} D_{a_{i}}
$$

Proof. Since G is an edge regular intuitionistic fuzzy graph, thus

$$
\begin{aligned}
\sum_{i=1}^{n} D_{a_{i} a_{i+1}} & =\left(\sum_{i=1}^{n} D_{\delta_{\mu}}\left(a_{i} a_{j}\right), \sum_{i=1}^{n} D_{\delta_{\lambda}}\left(a_{i} a_{j}\right)\right), \\
\sum_{i=1}^{n} D_{\delta_{\mu}}\left(a_{i} a_{i+1}\right) & =D_{\delta \mu}\left(a_{1} a_{2}\right)+D_{\delta \mu}\left(a_{2} a_{3}\right)+\cdots+D_{\delta \mu}\left(a_{n} a_{1}\right),
\end{aligned}
$$

since

$$
a_{n+1}=a_{1}=D_{\delta \mu}\left(a_{1}\right)+D_{\delta \mu}\left(a_{2}\right)-2 \gamma_{\mu}\left(a_{1} a_{2}\right)+D_{\delta \mu}\left(a_{2}\right)+D_{\delta \mu}\left(a_{3}\right)
$$




$$
\begin{aligned}
& -2 \gamma_{\mu}\left(a_{2} a_{3}\right)+D_{\delta \mu}\left(a_{n}\right)+D_{\delta \mu}\left(a_{1}\right)-2 \gamma_{\mu}\left(a_{n} a_{1}\right) \\
= & 2 D_{\delta \mu}\left(a_{1}\right)+2 D_{\delta \mu}\left(a_{2}\right)+2 D_{\delta \mu}\left(a_{n}\right)-2\left(\gamma_{\mu}\left(a_{1} a_{2}\right)+\gamma_{\mu}\left(a_{2} a_{3}\right)+\gamma_{\mu}\left(a_{n} a_{1}\right)\right) \\
= & 2 \sum_{a_{i} \in V} D_{\delta_{\mu}}\left(a_{1}\right)+2 \sum_{i=1}^{n} \gamma_{\mu}\left(a_{i} a_{i+1}\right)-2 \sum_{i=1}^{n} \gamma_{\mu}\left(a_{i} a_{i+1}\right) \\
= & \sum_{a_{i} \in V} D_{\delta_{\mu}}\left(a_{i}\right) .
\end{aligned}
$$

Then,

$$
\sum_{i=1}^{n} D_{\delta_{\mu}}\left(a_{i} a_{i+1}\right)=\sum_{a_{i} \in V} D_{\delta_{\mu}}\left(a_{i}\right)
$$

Similarly,

$$
\sum_{i=1}^{n} D_{\delta_{\lambda}}\left(a_{i} a_{i+1}\right)=\sum_{a_{i} \in V} D_{\delta_{\lambda}}\left(a_{i}\right)
$$

Therefore,

$$
\sum_{i=1}^{n} D_{a_{i} a_{i+1}}=\left(\sum_{i=1}^{n} D_{\delta_{\mu}}\left(a_{i} a_{i+1}\right), \sum_{i=1}^{n} D_{\delta_{\lambda}}\left(a_{i} a_{i+1}\right)\right)=\sum_{a_{i} \in V} D_{a_{i}} .
$$

Lemma 3.16. If $\mathrm{G}$ is an edge regular intuitionistic fuzzy graph on $\mathrm{G}^{*}$, then

$$
\sum_{a_{i} a_{j} \in E}^{n} D_{a_{i} a_{j}}=\left(\sum_{a_{i} a_{j} \in E}^{n} D_{G^{*}}\left(a_{i} a_{j}\right) \gamma_{\mu}\left(a_{i} a_{j}\right), \sum_{a_{i} a_{j} \in E}^{n} D_{G^{*}}\left(a_{i} a_{j}\right) \gamma_{\lambda}\left(a_{i} a_{j}\right)\right) \text {. }
$$

Since,

$$
D_{G}^{*}\left(a_{i} a_{j}\right)=D_{G}^{*}\left(a_{i}\right)+D_{G}^{*}\left(a_{j}\right)-2, \forall a_{i} a_{j} \in E .
$$

Proof. Straight forward.

Proposition 3.17. If $G$ is an edge regular intuitionistic fuzzy graph on a r-regular $G^{*}$, then

$$
\sum_{a_{i} a_{j} \in E} D_{a_{i} a_{i+1}}=\left((r-1) \sum_{a_{i}} D_{\delta_{\mu}}\left(a_{i}\right),(r-1) \sum_{a_{i}} D_{\delta_{\lambda}}\left(a_{i}\right)\right) .
$$

Proof. By Lemma 3.16 we obtain

$$
\begin{aligned}
\sum D_{a_{i} a_{j}} & =\left(\sum_{a_{i} a_{j} \in E} D_{G^{*}}\left(a_{i} a_{j}\right) \gamma_{\mu}\left(a_{i} a_{j}\right), \sum_{a_{i} a_{j} \in E} D_{G^{*}}\left(a_{i} a_{j}\right) \gamma_{\lambda}\left(a_{i} a_{j}\right)\right) \\
& =\left(\sum\left(D_{G^{*}}\left(a_{i}\right)+D_{G^{*}}\left(a_{j}\right)-2\right) \gamma_{\mu}\left(a_{i} a_{j}\right), \sum\left(D_{G^{*}}\left(a_{i}\right)+D_{G^{*}}\left(a_{j}\right)-2\right) \gamma_{\lambda}\left(a_{i} a_{j}\right)\right) .
\end{aligned}
$$

We know $G^{*}$ is a regular then the degree of every vertex in $G^{*}$ is $r$, this means that $D_{G} *\left(a_{i}\right)=r$ and hence

$$
\begin{aligned}
\sum D_{a_{i} a_{j}} & =\left((r+r-2) \sum \gamma_{\mu}\left(a_{i} a_{j}\right),(r+r-2) \sum \gamma_{\lambda}\left(a_{i} a_{j}\right)\right) \\
& =\left(2(r-1) \sum \gamma_{\mu}\left(a_{i} a_{j}\right), 2(r-1) \sum \gamma_{\lambda}\left(a_{i} a_{j}\right)\right) \\
& =\left((r-1) \sum D_{\delta_{\mu}}\left(a_{i}\right),(r-1) \sum D_{\delta_{\lambda}}\left(a_{i}\right)\right) .
\end{aligned}
$$


Corollary 3.18.

$$
\sum T_{D}\left(a_{i} a_{j}\right)=\left(\sum D_{G^{*}}\left(a_{i} a_{j}\right) \gamma_{\mu}\left(a_{i} a_{j}\right)+\sum \gamma_{\mu}\left(a_{i} a_{j}\right), \sum D_{G^{*}}\left(a_{i} a_{j}\right) \gamma_{\lambda}\left(a_{i} a_{j}\right)+\sum \gamma_{\lambda}\left(a_{i} a_{j}\right)\right),
$$

where $G$ is an edge regular intuitionistic fuzzy graph on $G^{*}$.

Proof.

$$
\begin{aligned}
\sum \mathrm{T}_{\mathrm{D}}\left(\mathrm{a}_{i} \mathrm{a}_{j}\right) & =\left(\sum \mathrm{T}_{\mathrm{D}} \delta_{\mu}\left(a_{i} a_{j}\right), \sum \mathrm{T}_{\mathrm{D}} \delta_{\lambda}\left(a_{i} a_{j}\right)\right) \\
& =\left(\sum \mathrm{T}_{\mathrm{D}} \delta_{\mu}\left(a_{i} a_{j}\right)+\gamma_{\mu}\left(a_{i} a_{j}\right), \sum \mathrm{T}_{\mathrm{D}} \delta_{\mu}\left(a_{i} a_{j}\right)+\gamma_{\lambda}\left(a_{i} a_{j}\right)\right) \\
& =\left(\sum \mathrm{D}_{\delta_{\mu}}\left(a_{i} a_{j}\right)+\sum \gamma_{\mu}\left(a_{i} a_{j}\right), \sum D_{\delta_{\lambda}}\left(a_{i} a_{j}\right)+\sum \gamma_{\lambda}\left(a_{i} a_{j}\right)\right) .
\end{aligned}
$$

By Lemma 3.16, we get

$$
\sum T_{D}\left(a_{i} a_{j}\right)=\left(\sum D_{G^{*}}\left(a_{i} a_{j}\right) \gamma_{\mu}\left(a_{i} a_{j}\right)+\sum \gamma_{\mu}\left(a_{i} a_{j}\right), \sum D_{G^{*}}\left(a_{i} a_{j}\right) \gamma_{\lambda}\left(a_{i} a_{j}\right)+\sum \gamma_{\lambda}\left(a_{i} a_{j}\right)\right) .
$$

Definition 3.19. An intuitionistic fuzzy graph $G$ is said to be strongly regular when:

1. $G$ is $\left(r_{1}, r_{2}\right)$-regular intuitionistic fuzzy graph;

2. the sum of member value and non-membership values of the common neighborhood vertices of any pair of adjacent vertices and non adjacent vertices $a_{i}, a_{j}$ of $G$ has the same weight and its denoted by $\alpha=\left(\alpha_{1}, \alpha_{2}\right), \beta=\left(\beta_{1}, \beta_{2}\right)$, respectively. A strongly intuitionistic fuzzy graph $G$ is denoted by $\mathrm{SI}_{\mathrm{G}}=(\mathrm{r}, \alpha, \beta)$.

Theorem 3.20. Let $G$ be a complete intuitionistic fuzzy graph such that $\left(\delta_{\mu}, \delta_{\lambda}\right)$ and $\left(\gamma_{\mu}, \gamma_{\lambda}\right)$ as a constant functions, then $G$ is a strongly regular intuitionistic fuzzy graph.

Proof. Since that $\left(\delta_{\mu}, \delta_{\lambda}\right)$ and $\left(\gamma_{\mu}, \gamma_{\lambda}\right)$ are constant functions, then

$$
\delta_{\mu}\left(a_{\mathfrak{i}}\right)=k, \delta_{\lambda}\left(a_{\mathfrak{i}}\right)=s \quad \text { and } \quad \delta_{\mu}\left(a_{\mathfrak{i}} a_{\mathfrak{j}}\right)=d_{1}, \delta_{\lambda}\left(a_{i} a_{\mathfrak{j}}\right)=d_{2},
$$

such that $k, s, d_{1}, d_{2}$ are constants, and $G$ is complete, then

$$
D_{a_{i} a_{j}}=\left((n-1) d_{1},(n-1) d_{2}\right),
$$

therefore $G$ is

$$
\left(\sum \delta_{\mu}\left(a_{i} a_{j}\right), \sum \delta_{\lambda}\left(a_{i} a_{j}\right)\right)=\left(D_{\delta \mu}\left(a_{i} a_{j}\right), D_{\delta \lambda}\left(a_{i} a_{j}\right)\right)=\left((n-1) d_{1},(n-1) d_{2}\right),
$$

regular intuitionistic fuzzy graph, where $\mathrm{n}$ is the number of vertices. On the other hand, the sum of membership values and non membership of common neighborhood vertices of any pair of adjacent vertices $\alpha=((n-2) k,(n-2) s)$ are equal, and the sum of membership values and non membership values of common neighborhood vertices of any pair of non adjacent vertices $\beta=0$ are the same.

Theorem 3.21. If $\mathrm{G}$ is a strongly regular intuitionistic fuzzy graph which is strong, then $\mathrm{G}^{\mathrm{c}}$ is $a\left(\mathrm{r}_{1}, \mathrm{r}_{2}\right)$ regular.

Proof. We know G is strong, then

$$
\gamma_{\mu}^{c}\left(a_{i} a_{j}\right)=\left\{\begin{array}{ll}
0, & a_{i} a_{j} \in \gamma, \\
\min \left\{\delta_{\mu}\left(a_{i}\right), \delta_{\mu}\left(a_{j}\right)\right\}, & a_{i} a_{j} \notin \gamma,
\end{array} \quad \gamma_{\lambda}^{c}\left(a_{i} a_{j}\right)= \begin{cases}0, & a_{i} a_{j} \in \gamma, \\
\max \left\{\delta_{\lambda}\left(a_{i}\right), \delta_{\lambda}\left(a_{j}\right)\right\}, & a_{i} a_{j} \notin \gamma .\end{cases}\right.
$$

Also

$$
D_{G}{ }^{c}\left(a_{i}\right)=\left(\mu_{D G}{ }^{c}\left(a_{i}\right), \lambda_{D G}{ }^{c}\left(a_{i}\right)\right),
$$


such that

$$
\begin{aligned}
& \mu_{D G}^{c}\left(a_{i}\right)=\sum_{a_{i} \neq a_{j}} \gamma_{\mu}^{c}\left(a_{i} a_{j}\right)=\sum_{a_{i} \neq a_{j}} \min \left(\delta_{\mu}^{c}\left(a_{i}\right), \delta_{\mu}^{c}\left(a_{j}\right)\right)=r_{1}, \\
& \mu_{D G}{ }^{c}\left(a_{i}\right)=\sum_{a_{i} \neq a_{j}} \gamma_{\lambda}^{c}\left(a_{i} a_{j}\right)=\sum_{a_{i} \neq a_{j}} \min \left(\delta_{\lambda}^{c}\left(a_{i}\right), \delta_{\lambda}^{c}\left(a_{j}\right)\right)=r_{2},
\end{aligned}
$$

for all $a_{i} \in V$. Thus, $D_{a_{i} a_{j}}=\left(r_{1}, r_{2}\right)$. Hence $G^{c}$ is $\left(r_{1}, r_{2}\right)$ regular intuitionistic fuzzy graph.

\section{Conclusion}

The models of graph are used in wide application in many area of computer science, mathematical models of social sciences. These graph models need to incorporate more structure than simply the adjacency between vertices. In the discussion of set behavior, it is observed that certain people can influence thinking of others. Each element of a set is represented by a node. There is a directed path from node $a_{i}$ to node $a_{j}$ when the member represented by node $a_{i}$ influence the node represented by node $a_{j}$.

In any social set all the nodes can never be members of the group always. Any node can be removed from the set at any time if his/her activity is against the set.

Each node of the set is represented by a vertex and every vertex has two values; the first value represents the power of the node in the set which means how much it posses to control the set, the second value represents the power of the node in the set when it become removed itself from the set. Each path has also two values such that the first component represents the influence by the first node over the second node when the first node is element of the set. The second component represent the influence by the first node over the second node when the first node is non-member of the set.

Any different fuzzy graph needs large data for training to be able to help in decision making technology and science. The new style which is generalized in this research is based on the pattern of unique cases that can help us to make a better choice in the contrast to the established solutions of fuzzy graph.

\section{References}

[1] K. T. Atanassov, Intuitionistic fuzzy sets, Fuzzy Sets and Systems, 20 (1986), 87-96. 1

[2] A. Bozhenyuk, M. Knyazeva, I. Rozenberg, Algorithm for finding domination set in intuitionistic fuzzy graph, Proceedings of the 11th Conference of the European Society for Fuzzy Logic and Technology (EUSFLAT 2019), Atlantis Press, 1 (2019), 72-76. 1

[3] B. Davvaz, N. Jan, T. Mahmood, K. Ullah, Intuitionistic fuzzy graphs of nth type with applications, J. Intell. Fuzzy Syst., 36 (2019), 3923-3932.

[4] P. J. Jayalakshmi, C. V. R. Harinarayanan, P. Muthuraj, Total strong (weak) domination intuitionistic fuzzy graph, Adv. Theor. Appl. Math., 11 (2016), 203-212. 1

[5] M. G. Karunambigai, O. K. Kalaivani, Matrix representation of intuitionistic fuzzy graph, Int. J. Sci. Res., 6 (2016), 520-537. 1

[6] M. O. Massa'deh, A. K. Baareh, Some contribution on isomorphic fuzzy graphs, Adv. Appl. Discrete Math., 11 (2013), 199-206. 1

[7] M. O. Massa'deh, N. K. Gharaibeh, Some properties on fuzzy graph, Adv. Fuzzy Math., 6 (2011), 245-252. 1

[8] S. N. Mishra, A. Pal, Regular interval-valued intuitionistic fuzzy graphs, J. Inform. Math. Sci., 9 (2017), 609-621. 1

[9] S. Y. Mohamed, R. J. Hussain, Isomorphism on irregular intuitionistic fuzzy graph and it's complements, IOSR J. Math., 10 (2014), 149-154. 1

[10] S. I. Mohideen, A. N. Gani, B. F. Kani, C. Yasmin, Properties of operations on regular intuitionistic fuzzy graphs, Int. J. Eng. Sci. Comput., 6 (2016), 3779-3783. 1

[11] S. R. Narayanan, N. R. S. Maheswari, Strongly edge irregular interval-valued fuzzy graphs, Int. J. Math. Arch., 7 (2016), 192-199. 1

[12] K. R. S. Narayan, M. S. Sunitha, Connectivity in a fuzzy graph and its complement, Gen. Math. Notes, 9 (2012), $38-43$. $1,2.1,2.2$

[13] R. Parvathi, M. G. Karunambigai, Intuitionistic fuzzy graphs, In: Reusch B. Computational Intelligence, Theory and Applications, Springer, 20 (2006), 139-150. 1, 2.7, 2.8, 2.9, 2.10

[14] T. Pathinathan, J. J. Rosline, Vertex degree of cartesian product of intuitionistic fuzzy graphs, Int. J. Sci. Eng. Res., 5 (2014), 224-227. 1 
[15] C. Ramprasad, N. Srinivasarao, S. Satyanarayana, A study on interval-valued fuzzy graphs, Comput. Sci. Telecommun., 50 (2016), 60-72. 1

[16] A. Rosenfeld, Fuzzy graphs, in: Fuzzy sets and their applications to cognitive and decision processes, Academic Press, (1975), 77-95. 1, 2.4, 2.5, 2.6

[17] S. Samanta, M. Akram, M. Pal, m-step fuzzy competition graphs, J. Appl. Math. Comput., 47 (2015), 461-472. 1

[18] T. Sunny, S. M. Jose, Modular and homomorphic products on interval - Valued intuitionistic fuzzy graphs, Int. J. Pure Appl. Math., 118 (2018), 257-276. 1

[19] N. Yaqoob, M. Gulistan, S. Kadry, H. A. Wahab, Complex intuitionistic fuzzy graphs with application in cellular network provider companies, Math., 7 (2019), 18 pages. 1

[20] L. A. Zadeh, Fuzzy sets, Inf. Control, 8 (1965), 338-353. 1, 2.3 